\begin{tabular}{|c|c|}
\hline & $\begin{array}{l}\text { International Journal of Trend in Scientific } \\
\text { Research and Development (IJTSRD) }\end{array}$ \\
\hline 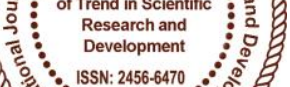 & International Open Access Journal \\
\hline 00 & ISSN No: 2456 - 6470 | www.ijtsrd.com | Volume - 2 | Issue -4 \\
\hline
\end{tabular}

\title{
New Adaptive Cooperative-MIMO for LTE
}

\author{
Sukhreet Kaur, Dr. Amita Soni \\ Punjab Engineering College, Chandigarh, India
}

Abstract-- Multiple Input Multiple Output (MIMO) systems have been widely used in an area of wireless cellular communication system, providing the both increased capacity and reliability. However, the use of multiple antennas in mobile terminals may not be very practical due to limited space and other implementation issues. In this paper, cooperative MIMO has been used in a way to optimise the implementation and working of conventional MIMO systems in terms of BER and Spectral Efficiency while maintaining a minimal number of antennas on each handset. Cooperative MIMO with V-BLAST transmission over Rayleigh flat fading channels and amplify and forward protocol with one relay node for modulation techniques like BPSK, QPSK, QAM using various decoding techniques has been analysed. Decoding algorithms like ZF, MMSE and ML have been analysed with respect to their BER performances. Since, there is throughput loss in cooperative MIMO due to extra resources required for relaying, adaptive modulation has been used with $\mathrm{C}$ MIMO to meet the demands for high data rates in Long Term Evolution Network.

Keywords-- Amplify and Forward (AF), MRC (Maximal Ratio Combining), V-BLAST (Vertical Bell Laboratories Layered Space-Time), Zero Forcing $(\mathrm{ZF})$, Minimum Mean Square Error (MMSE), Maximum Likelihood (ML), BER, Spectral Efficiency (SE), BPSK, QPSK

\section{INTRODUCTION}

MIMO System has emerged as one of the powerful technique in the field of wireless communication improving reliability to a great extent. However, the antennas on a MIMO device should have sufficient spacing for uncorrelated fading. If the spacing between antennas reduces, independent fading cannot be achieved for each pair of antennas and the efficiency of MIMO will be degraded. Moreover, real-time implementation of multiple antennas in a small device like mobile is very unreal and cost ineffective because of extra RF hardware.

To overcome the problems faced in MIMO Systems, a new cooperative MIMO is a practical solution which allows a source node to communicate with destination node with the help of wireless relays which are capable of achieving spatial diversity in a distributed manner.

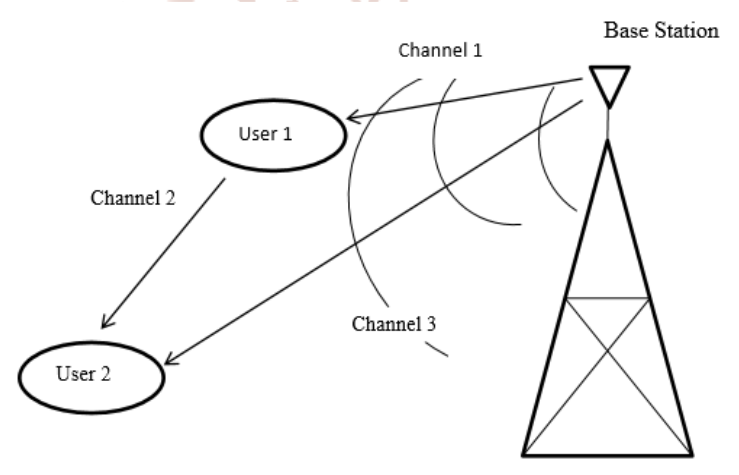

Figure 1: Cooperative Communication

In Figure 1, it is shown that two mobile users are communicating with base station. Because of the inherent broadcast nature of wireless devices at base station, it is quite possible that one user may receive the information intended to receive by another user and so it can help forward some version of received information to the intended user. Since fading channels are statistically independent to both the users, so receive diversity is generated.

Because of this diversity, the negative effects of shadowing and small-scale fading can be reduced as well error probabilities are also decreased. However, one main challenge in cooperative MIMO is throughput loss due to extra resources required for relaying. So, in order to nullify this effect, adaptive modulation is used. 
This paper analyses the BER and Spectral Efficiency of the cooperative MIMO system aided by adaptive modulation scheme over the Rayleigh fading channel under perfect CSI.

\section{RELATED WORK}

Many researches have been done focussing on Adaptive modulation in Cooperative MIMO finding a way to improve spectral efficiency. In [9], a new joint adaptive modulation and MIMO transmission algorithm has been studied under imperfect CSI. In [10], various AM schemes have been studied in MIMO-OSTBC to analyse the performance of Variable-power Adaptive Modulation with imperfect CSI. In [11], adaptive modulation for OFDM system over frequency selective fast fading channel was studied. The performance analysis of adaptive modulation in MIMO system-based Imperfect CSI showed that significant gains can be achieved with rate adaptation even with imperfect CSI in [12]. A novel hybrid Cooperative AMC mechanism is suggested by authors in [14] for improving hybrid link quality. Therefore, adapting constellation size of transmitted signal to changing channel conditions can track the wireless channel properties and thus sending more bits in good channel state and less bits in poor channel state can help increasing spectral efficiency.
2. Minimum Mean Square Error (MMSE): This performs better than the ZF under noisy conditions. It is not able to discard ISI like ZF but however, it reduces the total noise power received at the receiver side. The MMSE filter is calculated by minimizing the mean-square error (MSE) as: WMMSE $=(\mathrm{HTH}+$ $\sigma 2 \mathrm{IN})-1 \mathrm{HT}$ where $\sigma$ is Ratio of received signal power to noise power and IN is Identity matrix.

3. Maximum Likelihood (ML): The decision is taken according to the minimum Euclidean distance between the received signal vector and the product of all possible transmitted signal vectors with channel $\mathrm{H}$, and one with the least distance is found.

\section{COMBINING TECHNIQUES}

Incoming signals with same information have to be combined if more than one in number. Some of these combining techniques are:

Maximum Ratio Combining (MRC), Equal Gain Combiner, Fixed Ratio Combining, Signal to Noise Ratio Combining, Estimated SNR Combining In this paper, MRC scheme is used. In MRC, each radio channel is weighted with the complex conjugate of the respective channel gain assuming that channels' phase shift and attenuation is perfectly known at the receiver.

VI. COOPERATIVE MIMO OVER RAYLEIGH FADING CHANNEL SIMULATION MODEL

Few common relaying protocols are:

1. Amplify and Forward - In AF, received signal is scaled at relay node and transmitted to end user node after amplification.

2. Decode and Forward - In DF, the received signal is first decoded and then re-encoded. So, there is no amplified noise in received signal at end node.

3. Compress and Forward - In CF, a signal is quantized and compressed by relay and then forwarded to user node.

\section{DETECTION TECHNIQUES FOR MIMO SYSTEM}

Objective of the detection - to differentiate the transmitted signals on the basis of observation of the received signal vector and the channel matrix.

1. Zero-Forcing: $Z F$ nullifies the interference by WZF matrix, which is the pseudo inverse of channel matrix $\mathrm{H}$ i.e. $\mathrm{WZF}=(\mathbf{H T H})-1 \mathrm{HT}$.

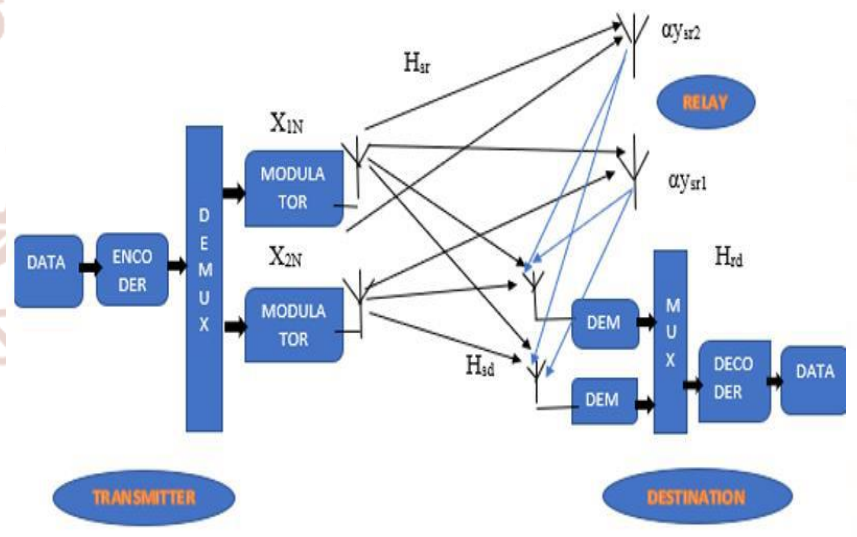

Figure 2: System Model of Cooperative MIMO

To evaluate the performance of Cooperative MIMO, all nodes are placed at equal distance with $\mathrm{Ns}=\mathrm{Nr}=$ $\mathrm{Nd}=2$ as shown in Fig 2 where $\mathrm{Ns}, \mathrm{Nr}, \mathrm{Nd}$ are number of antennas at source, relay and destination respectively such that $\mathrm{Nd} \geq \mathrm{Ns}=\mathrm{Nr}$ in order to separate and detect the $\mathrm{x}$ transmitted signals. Thus, a block fading half-duplex channel model with $\mathrm{N}$ fading blocks is assumed here, with L symbol periods in each 
block. On each block, Fading is assumed to be flat and constant but independently Rayleigh distributed on different blocks. Further, Average power is fixed per user due to uniform energy distribution with an assumption of perfect CSI available at the receiver side only.

There are two phases in which communication occurs. In phase one, the signal transmitted by source undergoes fading and is sent to relay. In phase two, depending upon the relay protocol, transceiver complexity, relative channel conditions and also user location, relay cooperate with the destination node. In this paper, Amplify and Forward protocol with variable relay gain is considered.

The equivalent cooperative $\mathrm{MIMO} \mathrm{NdNr} \times \mathrm{Ns}(4 \times 2)$ with $\mathrm{NdNr}$ (2) virtual receiving antennas representation for the proposed MIMO system using relays is given as:

$$
\begin{aligned}
& {\left[\begin{array}{l}
y_{d, 1 n l} \\
y_{d, 2 n l}
\end{array}\right]=\underbrace{\left[\begin{array}{c}
\sqrt{E s} H_{s d n l} \\
\frac{E s}{4} H_{r d n l} \propto_{n l} H_{s r n l}
\end{array}\right]}_{H_{\text {eqnl }}} x_{n l}+} \\
& {\left[\begin{array}{ccc}
I_{N_{S} N_{r}} & 0 & 0 \\
0 & \sqrt{\frac{E_{s}}{2}} H_{r d n l} \propto_{n l} & I_{N_{S} N_{r}}
\end{array}\right]\left[\begin{array}{l}
n_{s d n l} \\
n_{s r n l} \\
n_{r d n l}
\end{array}\right]}
\end{aligned}
$$

Where $\mathrm{yd}, 1 \mathrm{nl}$ and $\mathrm{yd}, 2 \mathrm{nl}$ are the received signals at the user end during the phase one and phase two respectively, Es is the average power per antenna of each symbol at the source. Hsrnl and Hsdnl denotes the nth block fading channel matrix of source-relay and source-destination link respectively. The vectors nsrnl and nsdnl are the noise due to source transmit the signal to relay and destination respectively. Index notation (nl) is used to emphasize more on the block fading nature of channel. $\propto$ nl is a gain factor of variable gain relay. The transmit signal from relay is:

$\mathrm{xrnl}=\alpha$ nlyrnl (2)

Therefore, the equivalent channel matrix for relayed link is:

Heqrsdnl $=$ Fnl $=$ HrdnlHsrnl (3)

While equivalent noise at receiver side is:

neqrsdnl $=$ Hrdnl $\alpha$ l nsrnl $+\operatorname{nrdnl}(4)$

After these two phases of cooperative transmission, the signals received at destination will be combined using one of the combining techniques discussed above and as the two networks source-destination and source-relay-destination links are different, so independent combining is done and then estimated values are summed up to get the final results.

\section{COOPERATION BASED ON V-BLAST TRANSMISSION}

Cooperative VBLAST transmission achieves high spectral efficiency by spatially multiplexing the coded or uncoded symbols transmitted by source in first phase over the fading channel. The received symbols are modified by relay and forwarded to destination in second phase. Using MRC technique, the total receive signal at the destination is given as follows:

ynl = HHsdnl yd,1 nl + HeqrsdnlH yd,2 nl (5)

In order to detect the transmitted signal $\mathrm{x}$, put $\mathrm{y}$ in $\mathrm{ZF}$, MMSE and ML detection scheme as $\mathrm{y}$ is in $\mathrm{y}=\mathrm{Hx}+$ $\mathrm{n}$ form.

\section{ADAPTIVE MODULATION IN COOPERATIVE MIMO}

The basic purpose of adaptive modulation is to apply the modulation based on CSI (Channel State Information) available. When the channel is in good state, higher order modulation scheme is used, otherwise lower order scheme is preferred. The various parameters that can used to indicate the CSI are SNR (Signal to Noise Ratio), SINR (Signal to Noise Ratio plus Interference), PERs (Packet Error Rates) or BER (Bit error Rate). In this paper, the post detection of SNR at the output of ZF, MMSE and ML detectors is used as a metric for CSI as it directly measures the performance of communication system. This post SNR will correspond to BER information for each mode candidate and then threshold SNR is selected depending on the mode that yields the highest throughput remaining with BER constraints.

Since exact post SNR detection is impossible, it can be estimated by sending pilot sequence through the channel to have the knowledge of current CSI. In this paper, it is assumed that CSI is known at receiver terminal.

Adaptation based SNR in assisted cooperative AF MIMO with VBLAST transmission

In order to select the suitable modulation scheme based on the specified BER target, threshold SNR has to be determined at the output of ZF, MMSE and ML detectors for which it yields maximum throughput. For that, post SNR equations has to be derived at the output of these given detectors considering all direct and non-direct links with V-BLAST transmission at receiver only. In addition, the data streams are sent 
independently to the relay and destination nodes and then collaboratively processed at destination to recover the original signal.

The equivalent channel matrices for direct and relayed links are given by Hsdnl and Fnl. For MRC technique the receive signal is: $\mathrm{ynl}=(\Sigma \sqrt{E s} \mathrm{Nr} i=1$ ( $\mid h i 1 \mathrm{dsnl}$ $|2+| h i 2 d s n l \mid 2)+E s 4 \propto n l(\mid$ fi1 $n l|2+|$ fi2 $n l \mid 2)$ $\mathrm{xnl}+\tilde{n} \mathrm{nl}(6)$

Where $F n l=H r d n l H s r n l$ and $\tilde{n} n l$ is the equivalent noise channel evaluated at the output of MRC that is

$\tilde{n} \mathrm{nl}=\mathrm{FH} \mathrm{nl}($ Hrdanl nsrnl + nrdnl $)+\mathrm{HH} \operatorname{sdnl}(7)$

In matrix form (6) can be written as:

$$
\left[\begin{array}{l}
y_{1 n l} \\
y_{2 n l}
\end{array}\right]=\sqrt{E} \underbrace{\left[\begin{array}{cc}
u_{11 n l} & 0 \\
0 & u_{22 n l}
\end{array}\right]}_{H_{n l}}\left[\begin{array}{l}
x_{1 n l} \\
x_{2 n l}
\end{array}\right]+\left[\begin{array}{l}
\tilde{n}_{11 n l} \\
\tilde{n}_{21 n l}
\end{array}\right]
$$

$\mathrm{u} 11 \mathrm{nl}=\sqrt{ } E(|\mathrm{~h} 11 \mathrm{dsn} 1| 2+|\mathrm{h} 21 \mathrm{dsn} 1| 2)+E s 4$ anl $(\mid \mathrm{f} 11$ $\mathrm{nl}|2+| \mathrm{f} 21 \mathrm{nl} \mid 2)$

$\mathrm{u} 22 \mathrm{nl}=\sqrt{E s}(|\mathrm{~h} 12 \mathrm{dsnl}| 2+|\mathrm{h} 22 \mathrm{dsnl}| 2)+E s 4$ anl ( $|\mathrm{f} 12 \mathrm{nl}| 2+|\mathrm{f} 22 \mathrm{nl}| 2)$

The covariance matrix of the equivalent noise (noise power) is

$$
\begin{aligned}
& \mathrm{E}\{\tilde{n} \mathrm{nl} \tilde{n} \mathrm{nlH}\}= \\
& N_{o}\left(\begin{array}{c}
\sum_{i=1}^{N_{r}}\left|f_{i 1 n l}\right|^{2}\left|f_{i 2 n l}\right|^{2}\left(1+\alpha^{2}{ }_{n l} \sum_{k=1}^{N_{r}}\left|h_{i k d r n l}\right|^{2}\right) \\
\sum_{i=1}^{N_{r}}\left|h_{i 1 d s n l}\right|^{2}-\left|h_{i 2 d s n l}\right|^{2}
\end{array}\right)
\end{aligned}
$$

The post SNR detection $j^{\text {th }}$ receive antenna at the output of ZF detector obtained from Eq. 12 given by: $\gamma_{j, n l}=$ $\frac{E_{S}}{N_{o}\left\|W_{i, M R C z f n l}\right\|_{F}^{2}\left(\left\|F_{i, n l}\right\|_{F}^{2}{ }^{+} \alpha^{2} n l\left\|F_{i, n l}\right\|_{F}^{2}{ }_{F}\left\|H_{i, r d n l}\right\|_{F}^{2}+\left\|H_{i, s d n l}\right\|_{F}^{2}\right.}$

\section{B. MMSE detection}

In this scheme, the transmitted signal xnl can be evaluated by minimizing the mean square error. MMSE detection matrix is given as

$$
W_{\text {MRCmmse } n l}=\left(H^{H}{ }_{n l} H_{n l}+N_{0} I_{N_{S}}\right)^{-1} H^{H}{ }_{n l}
$$

Multiplying Eq. 8 by Eq. 14, the estimate signal is

$$
\begin{aligned}
& {\left[\begin{array}{l}
y_{1 n l} \\
y_{2 n l}
\end{array}\right]=W_{\text {MRCmmse } n l} \underbrace{\left[\begin{array}{cc}
u_{11 n l} & 0 \\
0 & u_{22 n l}
\end{array}\right]}_{H n l}\left[\begin{array}{l}
x_{1 n l} \\
x_{2 n l}
\end{array}\right]+} \\
& W_{\text {MRCmmse } n l}\left[\begin{array}{l}
\tilde{n}_{11 n l} \\
\tilde{n}_{21 n l}
\end{array}\right]
\end{aligned}
$$

From Eq. 15, the post SNR detection for MMSE detection is given by:

$$
\begin{aligned}
& \gamma_{i, n l}= \\
& \frac{\left|W_{\text {MRCmmse nl }}\right|^{2}\left(\begin{array}{c}
E_{S}\left(\left|h_{i 1 d s n l}\right|^{2}+\left|h_{i 2 d s n l}\right|^{2}\right)+ \\
\left.\frac{E_{S}}{4} \alpha_{n l}\left|f_{i 1} n l\right|^{2}+\left|f_{i 2 n l}\right|^{2}\right)
\end{array}\right)^{2}}{N_{O}\left\|W_{i, M R C m m s e n l}\right\|_{F}^{2}\left(\begin{array}{c}
\left\|F_{i, n l}\right\|_{F}^{2}+\alpha^{2} n l\left\|H_{i, r d n l}\right\|_{F}^{2} \\
\left\|H_{i, s d n l}\right\|_{F}^{2}
\end{array}\right)}
\end{aligned}
$$

Where $\mathrm{E}\{$.$\} the expectation of \{$.$\} .$

\section{A. Zero Forcing detection}

The received signal in Eq. 8 can be seen as the sum of the required signal $x n l$ along with the noise. Extract $x \mathrm{nl}$ out of the received signal by estimate the values that minimize the least square of ynl $-\mathrm{Hnl} \hat{x} \mathrm{nl}$ and $\mathrm{ynl}$ - Gnlx̂nl.

$$
\mu_{\mathrm{MRCnl}}=\left\|\mathrm{y}_{\mathrm{nl}}-\mathrm{H}_{\mathrm{nl}} \hat{\chi}_{\mathrm{nl}}\right\|^{2}
$$

This is solved by multiplying by a pseudo inverse matrix:

$$
\begin{gathered}
W_{M R C z f n l}=\left(H_{n l}^{H} H_{n l}\right)^{-1} H^{H} \\
(11) \\
{\left[\begin{array}{c}
\hat{x}_{1 n l} \\
\hat{x}_{2 n l}
\end{array}\right]=\left[\begin{array}{l}
x_{1 n l} \\
x_{2 n l}
\end{array}\right]+\left(H^{H}{ }_{n l} H_{n l}\right)^{-1} H^{H}{ }_{n l}\left[\begin{array}{c}
\tilde{n}_{11 n l} \\
\tilde{n}_{21 n l}
\end{array}\right]}
\end{gathered}
$$

\section{ML detection}

In Maximum likelihood (ML), distance between the received signal vector in (8) and the product of all possible transmitted signal vectors with the given channel Hnl, Gnl, gives us the value of Euclidian distance and $x$ lwwith the min. distance metrics is chosen.

$$
\mathrm{d}^{2}\left(\mathrm{y}_{1 \mathrm{nl}}, \mathrm{u}_{11 \mathrm{nl}} \mathrm{x}_{1 \mathrm{nl}}\right)+\mathrm{d}^{2}\left(\mathrm{y}_{2 \mathrm{nl}}, \mathrm{u}_{22 \mathrm{nl}} \mathrm{x}_{1 \mathrm{nl}}\right)
$$

The estimated value of the transmitted signal vector $\mathrm{x}$ by ML detection is given as:

$$
\hat{x}_{n l}=\underset{\left\{\hat{x}_{1 n l}, \hat{x}_{2 n l}\right\}}{\operatorname{argmin}}\left\|y_{n l}-H_{n l} x_{n l}\right\|^{2}
$$


International Journal of Trend in Scientific Research and Development (IJTSRD) ISSN: 2456-6470

Where $\bar{C}$ is set of signal constellation symbol point possible value of $\left\{\hat{x}_{1 n l}, \hat{x}_{2 n l}\right\}$.

The post detection SNR $\gamma_{j, n l}$ MRC of the receive signal at the output of ML respectively from Eq. 19 given by

$$
\begin{aligned}
& \gamma_{i, n l}= \\
& \frac{\left(\begin{array}{c}
\left.E_{S}\left|h_{i 1 d s n l}\right|^{2}+\left|h_{i 2 d s n l}\right|^{2}\right)+ \\
\left.\frac{E_{s}}{4} \alpha_{n l}\left|f_{i 1 n l}\right|^{2}+\left|f_{i 2 n l}\right|^{2}\right)
\end{array}\right)^{2}}{N_{o}\left(\begin{array}{c}
\left(\mid f_{i 1} n l\right. \\
\left.\left.|+| f_{i 2 n l}\right|^{2}\right)\left(1+\alpha^{2} n l\right. \\
\left.\sum_{k=1}^{N r}\left|h_{i k d r n l}\right|^{2}\right)+
\end{array}\right)}
\end{aligned}
$$

The threshold for adaptive modulation has been derived above for ZF, MMSE and ML detectors by determining post SNR as a function of Channel State Information.

\section{SIMULATION RESULTS}

Simulation of adaptive modulation based cooperative MIMO is done in Matlab environment with all the parameters set according to LTE standard. For all simulations, classical relay model is considered and equal power is given to each node with independent identically distributed (i.i.d) Rayleigh fading channel matrix elements. Cooperative and Non- Cooperative transmissions are compared in terms of BER and Spectral Efficiency.

Table 1: Simulation Parameter

\begin{tabular}{|l|l|}
\hline Parameter & Type / Value \\
\hline Channel & Rayleigh flat fading \\
\hline $\begin{array}{l}\text { Transmit, receive and relay } \\
\text { antennas number }\end{array}$ & 2,2 and 2 respectively \\
\hline Transmit power & normalized at all nodes \\
\hline Modulation Scheme & $\begin{array}{l}\text { BPSK, QPSK,16 QAM and 64 } \\
\text { QAM }\end{array}$ \\
\hline Combining techniques & MRC \\
\hline Detectors & ZF, MMSE and ML \\
\hline MIMO scheme & $\begin{array}{l}\text { Spatial multiplexing with V- } \\
\text { BLAST }\end{array}$ \\
\hline Relay protocol & AF protocol \\
\hline Threshold BER or BERth & BER $\leq 10^{-2}$ \\
\hline Performance Metrics & BER and SE \\
\hline
\end{tabular}

Performance of $2 \times 2$ V-BLAST scheme with MRC

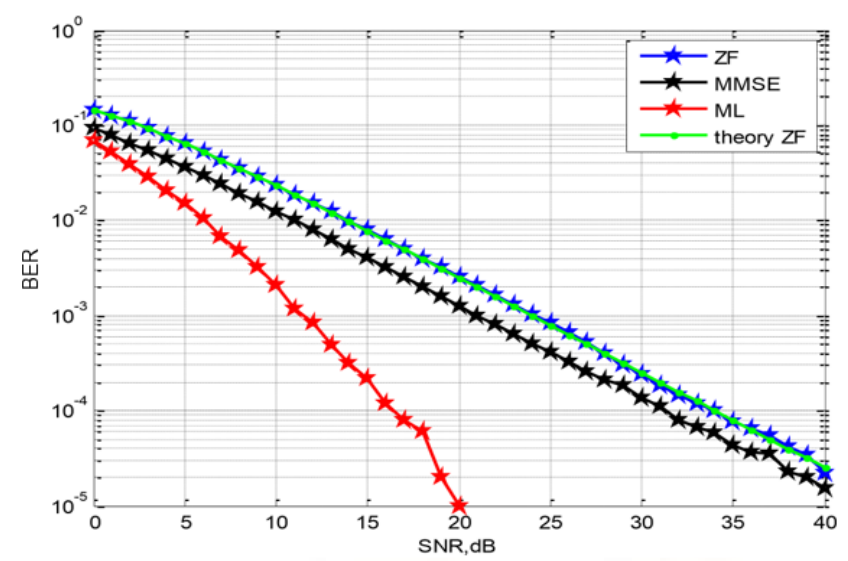

Figure 3: BER performance of $2 \times 2 \mathrm{~V}$-BLAST MIMO using BPSK modulation for different detection scheme

In Figure 3, it is seen that for a fixed BER of 10-3, SNR required for $\mathrm{ZF}, \mathrm{MMSE}$ and $\mathrm{ML}$ detection is approximately 24.5, 21.5 dB and $11.5 \mathrm{~dB}$ respectively. ZF and MMSE detectors showed almost same performance but ML has a better performance than these.

\section{$2 \times 2$ V-BLAST scheme (MRC) for MMSE detection}

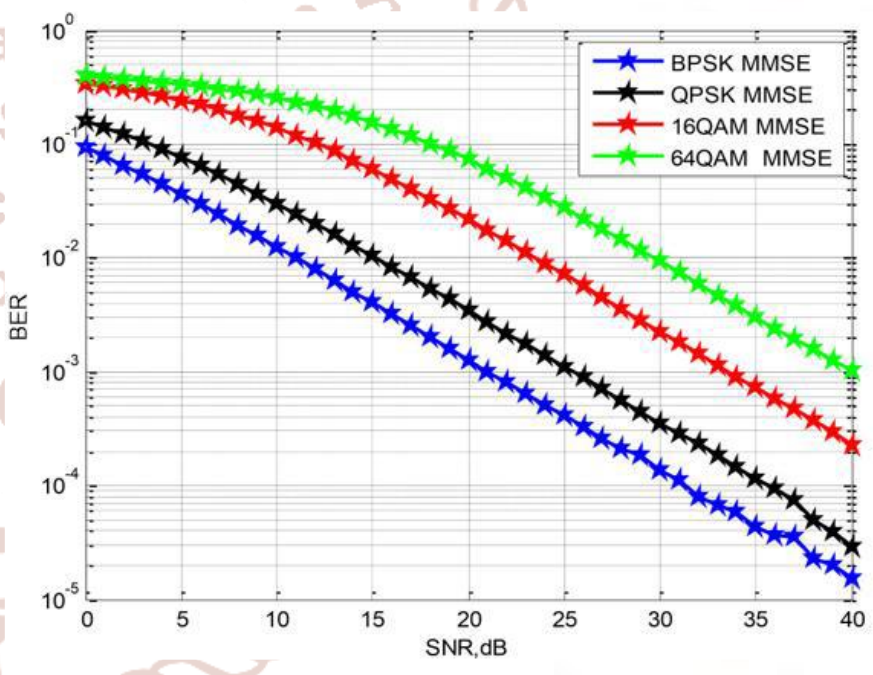

Figure 4: BER performance of $2 \times 2 \mathrm{~V}$-BLAST MIMO system using MMSE detection for different modulation

In Figure 4, it can be seen that a change in constellation size affects the BER performance a lot. A change in $M=6$ to $M=2$ indicates performance betterment close to $8.5 \mathrm{~dB}$ at a $\mathrm{BER}=10-3$ in $\mathrm{MIMO}$ VBLAST using MMSE detection. 
International Journal of Trend in Scientific Research and Development (IJTSRD) ISSN: 2456-6470

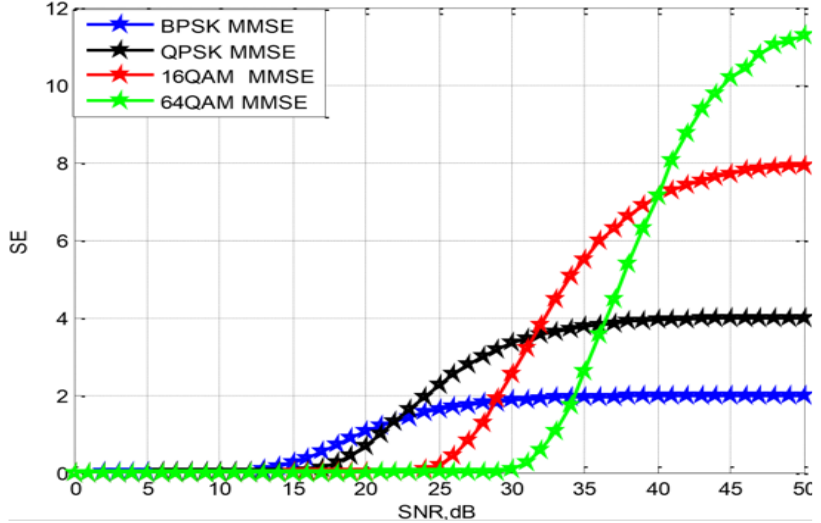

Figure 5: Spectral efficiency of $2 \times 2 \mathrm{~V}$-BLAST MIMO using MMSE detection for different modulation.

In Figure 5, Throughput curves are obtained for MMSE detection for different modulation techniques. Also, switching criterion is obtained corresponding to given modulation types for already set value of BER assuming it to be 10-2 so that realistic BER $\leq 10-2$.

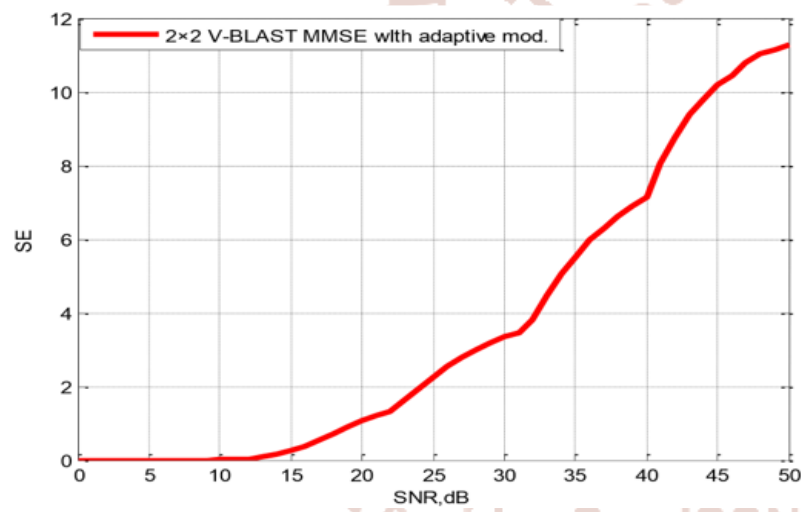

Figure 6: Spectral efficiency of adaptive modulation $2 \times 2$ V-BLAST MIMO using MMSE detection

In Figure 6, the simulation results of the adaptive modulation in $2 \times 2 \mathrm{~V}$-BLAST MIMO system for MMSE detection are presented. For different SNR ranges, we can select most suitable modulation type as given in the following Table 2 making the system adaptive.

Table 2: Probability of choosing different modulation type for MMSE detection

\begin{tabular}{|c|l|}
\hline SNR, dB, For BER $\leq 10^{-2}$ & Modulation Type \\
\hline$<11$ & No transmission \\
\hline$[11,22]$ & BPSK \\
\hline$[22,32]$ & QPSK \\
\hline$[32,40]$ & 16 QAM \\
\hline$>40$ & 64 QAM \\
\hline
\end{tabular}

Performance of $2 \times 2 \quad V$-BLAST scheme with MRC for $M L$ detection
Figure 7 and Figure 8 shows BER performance and throughput curves for 2 X 2 VBLAST MIMO system.

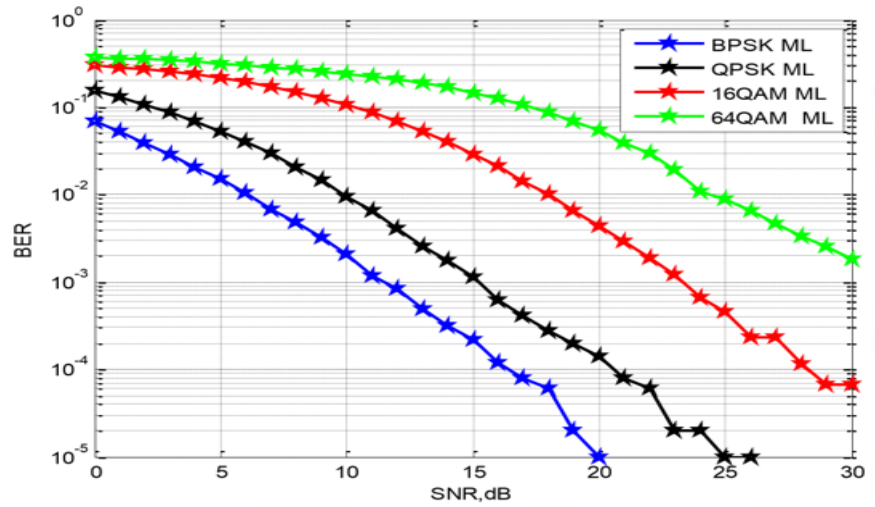

Figure 7: BER performance of $2 \times 2 \mathrm{~V}$-BLAST MIMO system using ML detection for different modulation

In Figure 7, for BER of 10-2, ML detection improves performance of MMSE by 10dB using QPSK.

From Figures 6 and 8, adaptive modulation MIMO system for MMSE and ML respectively, it can be seen that at SNR of $20 \mathrm{~dB}$ the ML has throughput improvement of $26.3 \%$ over MMSE detection. The MMSE detection technique has to gain $12 \mathrm{~dB}$ to get the same throughput

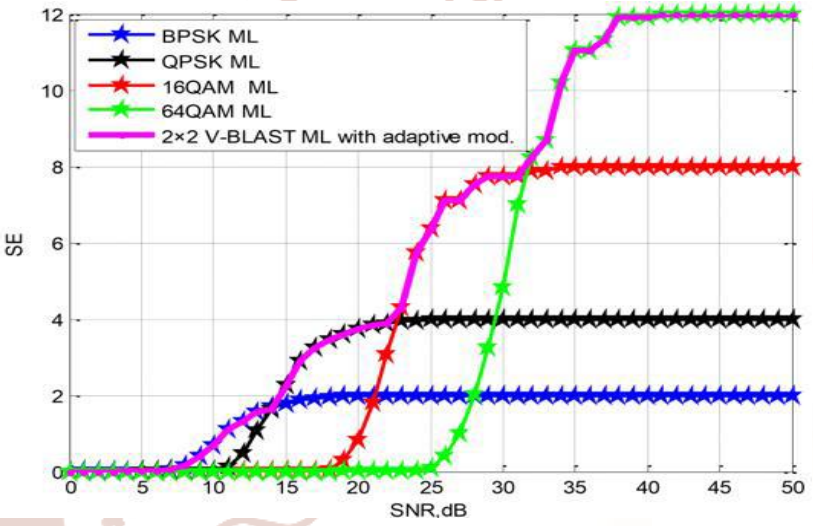

Figure 8: Spectral efficiency of adaptive modulation $2 \times 2$ V-BLAST MIMO using ML detection

Performance of V-BLAST scheme with MRC (Cooperative) 


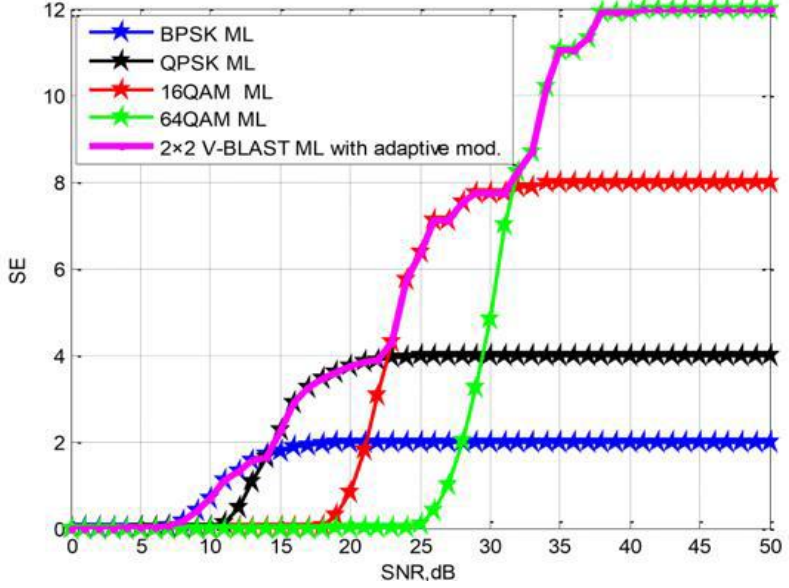

Figure 9: BER performance of cooperative diversity, $2 \times 2$ V-BLAST MIMO, and SISO with BPSK modulation.

Figure 9 shows BER performance of cooperative, $2 \mathrm{X} 2$ VBLAST MIMO, SISO with BPSK. It can be seen that simulated results for cooperative diversity are in accordance with the theoretically derived values. At BER of 10-3, increasing the no. of antennas from single to two gains $6 \mathrm{~dB}$ over SISO. Also, on adding one AF relay node in between gains $7 \mathrm{~dB}$ gain over non-cooperative VBLAST MIMO system at same BER. Simulated results came out from diversity of direct link and assistance link of relay.

\section{Performance of Cooperative $2 \times 2 \mathrm{~V}$-BLAST scheme with MRC for MMSE detection}

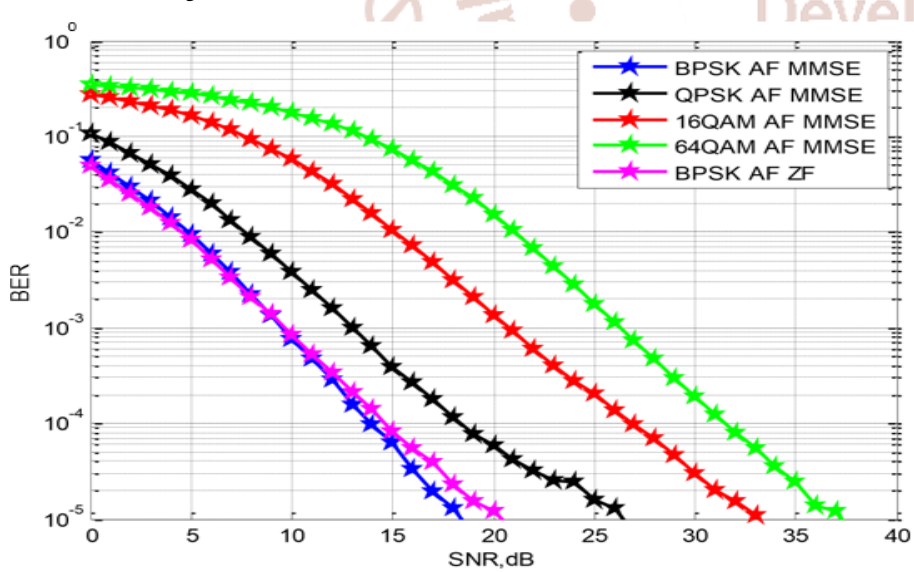

Figure 10: BER Performance of Cooperative $2 \times 2 \mathrm{~V}$ -

BLAST MIMO system using MMSE and ZF

detections for different modulation schemes

Figure 10 shows BER performance of AF 2X2 VBLAST MIMO for MMSE and ZF detections. It can be seen that $\mathrm{ZF}$ and MMSE have almost performance in the $\mathrm{SNR} \leq 18 \mathrm{~dB}$ range for BPSK modulation.

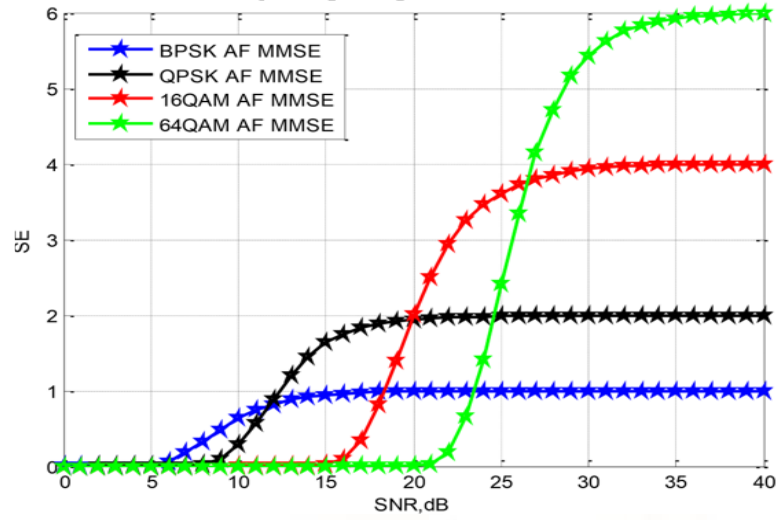

Figure 11: Throughput performance Cooperative $2 \times 2$ V-BLAST system using MMSE detection for different modulation

Figure 11 indicates the performance of cooperative VBLAST MIMO for MMSE detection. It is clearly visible that SE saturates to half as compared to noncooperative results but this is due to half duplex transmission used. From Figure 5, a spectral efficiency of $3 \mathrm{bit} / \mathrm{sec}$ can be achieved with a SNR of about $31 \mathrm{~dB}$ with 16 QAM; for the same modulation this value is reduced to about $22 \mathrm{~dB}$ if Cooperative $2 \times 2 \mathrm{~V}$-BLAST MIMO with MMSE scheme is used as shows in Figure 11.

SNR Threshold values are obtained in Table 3 for Cooperative V-BLAST MIMO for MMSE detection scheme.

Table 3: SNR switching criterion of AF $2 \times 2 \mathrm{~V}$ BLAST MIMO system using MMSE detection

\begin{tabular}{|c|l|}
\hline SNR, $\mathrm{dB}$ (for $\mathrm{BER}_{\mathrm{m}} \leq 10^{-2}$ ) & Modulation Type \\
\hline$<5$ & No transmission \\
\hline$[5,12]$ & BPSK \\
\hline$[12,20]$ & QPSK \\
\hline$[20,27]$ & $16 \mathrm{QAM}$ \\
\hline$>27$ & $64 \mathrm{QAM}$ \\
\hline
\end{tabular}

Performance of Cooperative $2 \times 2 \quad \mathrm{~V}$-BLAST scheme with MRC for ML detection 
International Journal of Trend in Scientific Research and Development (IJTSRD) ISSN: 2456-6470

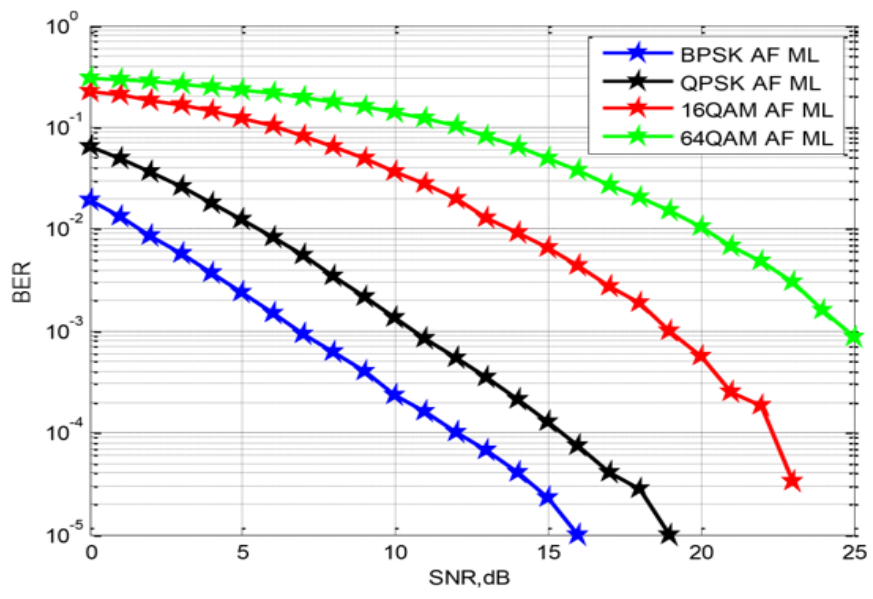

Figure 12: BER Performance of Cooperative $2 \times 2 \mathrm{~V}$ BLAST MIMO system using ML detection for different modulation scheme

Figure 12 shows that BER performance of Cooperative VBLAST MIMO system is better for ML detection than that of MMSE detection. It also shows that as the constellation size increases, performance decreases

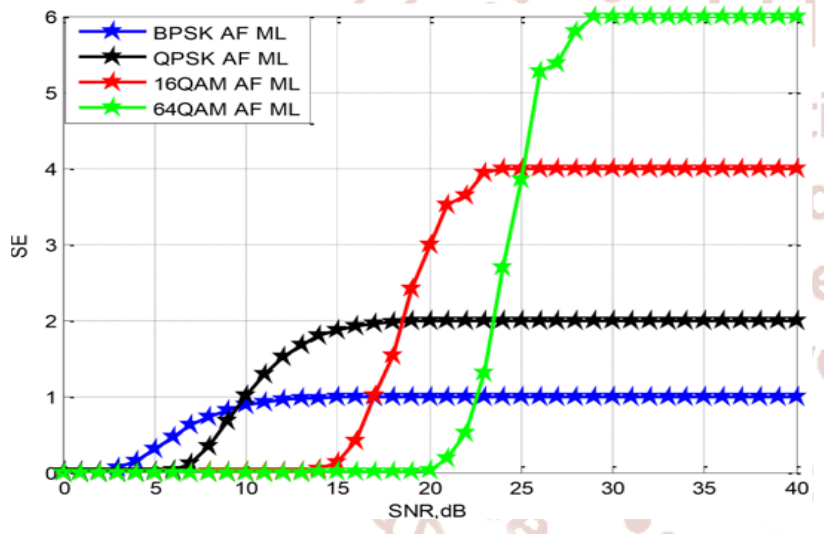

Figure 13: Throughput performance of Cooperative

$2 \times 2 \mathrm{~V}$-BLAST system using ML detection for different modulation

Figure 13 depicts the Throughput performance for ML detection of Figure 12, at target of BER $\leq 10-2$. In this figure, there is no modulation scheme that gives us performance below 10-2 at an SNR below $3 \mathrm{~dB}$.

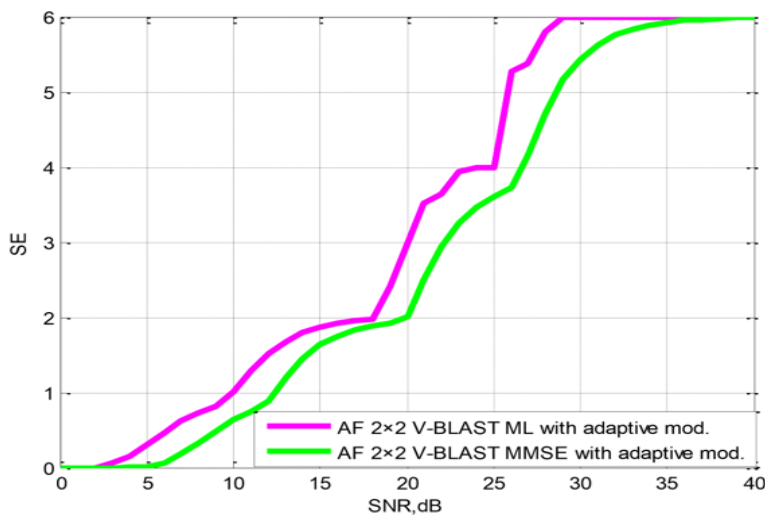

Figure 14: Throughput performance of adaptive modulation cooperative $2 \times 2 \mathrm{~V}$-BLAST MIMO using MMSE and ML detection.

Figure 14 shows the comparison between throughput performance of MMSE and ML detections. It can be seen that the ML Detection, in contrast to the MMSE Detection has improved spectral efficiency by approximately $2.5 \mathrm{~dB}$ at medium or low SNRs. However, for SNR greater than $35 \mathrm{~dB}$ the gap is approximately nil.

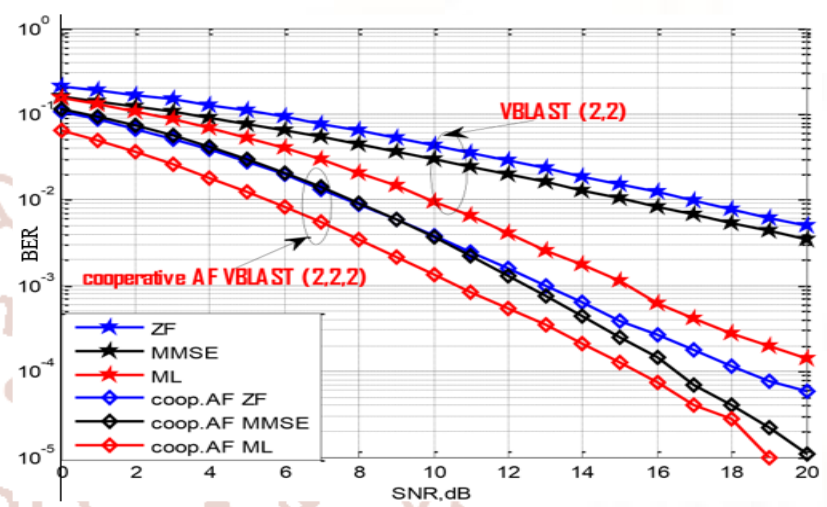

Figure 15: BER Performance curves of cooperative $2 \times 2$ V-BLAST MIMO system for different detectors (ML, ZF and MMSE).

Figure 15 shows the comparison of 2 X 2 VBLAST MIMO system with Cooperative AF 2 X 2 VBLAST MIMO system for all detection techniques. The result revealed that at BER of 10-2 there is approximately $1 \mathrm{~dB}$ SNR difference between the $\mathrm{ZF}$ and MMSE detectors in $2 \times 2$ V-BLAST MIMO system. Cooperative $2 \times 2$ VBLAST MIMO system shows the performance curves of these two detectors close to each other, especially when the SNR is less than $11 \mathrm{~dB}$. The cooperative $2 \times 2 \mathrm{~V}$-BLAST MIMO system with ML detection yields a gain of about $4.5 \mathrm{~dB}$ over the corresponding $2 \times 2 \mathrm{~V}$-BLAST MIMO system with ML detection at a BER of .

The SNR gains of cooperative MIMO using QPSK modulation over non-cooperative for ZF, MMSE and ML detections at a BER of 10-2 are listed in Table 4.

Table 4: SNR switching method of AF cooperative 2X2 V-BLAST MIMO system with MMSE detection 
International Journal of Trend in Scientific Research and Development (IJTSRD) ISSN: 2456-6470

\begin{tabular}{|c|c|c|c|c|c|c|c|c|c|}
\hline \multirow{3}{*}{$\begin{array}{l}\text { Mod } \\
\text { ulati } \\
\text { on } \\
\text { type }\end{array}$} & \multicolumn{6}{|c|}{ Systems } & \multirow{2}{*}{\multicolumn{3}{|c|}{$\begin{array}{c}\text { SNR Gains } \\
\text { Cooperative } \\
\text { MIMO }\end{array}$}} \\
\hline & \multicolumn{3}{|c|}{ MIMO } & \multicolumn{3}{|c|}{$\begin{array}{c}\text { Cooperative } \\
\text { MIMO }\end{array}$} & & & \\
\hline & $\mathrm{ZF}$ & $\begin{array}{c}\mathrm{MM} \\
\mathrm{SE}\end{array}$ & $\begin{array}{l}\mathrm{M} \\
\mathrm{L}\end{array}$ & $\mathrm{ZF}$ & $\begin{array}{c}\mathrm{MM} \\
\mathrm{SE}\end{array}$ & $\begin{array}{l}\mathrm{M} \\
\mathrm{L}\end{array}$ & $\mathrm{ZF}$ & $\begin{array}{c}\mathrm{MM} \\
\mathrm{SE}\end{array}$ & $\begin{array}{l}M \\
\mathrm{~L}\end{array}$ \\
\hline QPS & 16 & 15 & 10 & 8 & $8 \mathrm{~dB}$ & 5.5 & 8 & $7 \mathrm{~dB}$ & 4.5 \\
\hline $\mathrm{K}$ & $\mathrm{dB}$ & $\mathrm{dB}$ & $\mathrm{dB}$ & $\mathrm{dB}$ & & $\mathrm{dB}$ & $\mathrm{dB}$ & & dB \\
\hline
\end{tabular}

CONCLUSION

The simulation results of Cooperative and Noncooperative MIMO systems over Rayleigh fading channel for $\mathrm{ZF}, \mathrm{MMSE}$ and $\mathrm{ML}$ detections using MRC combining have been analysed along with adaptive modulation. Results have shown that cooperative VBLAST MIMO in contrast to noncooperative VBLAST MIMO system is capable of improved and better BER and SE performance at no penalty in complexity. ML detector proved to be optimal detector. Furthermore, adaptive modulation aided to select optimal modulation scheme in different SNR ranges achieving the highest SE with BER target set at $10-2$.

\section{References}

[1] P. K. Kharat And J. D.Gavade, "Cooperative Communication: New Trend In Wireless Communication," Int.J. Future Generation Commun. Networking, Vol. 6, Iss.5, Pp. 157-166, 2013

[2] S. Medhn, "Cooperative MIMO System for WiMAX Technology," MSc. thesis, Dept. Elect. Eng and Comp., University of Addis Ababa, Ethiopia, 2012.

[3] Moubachir Madani Fadoul, Mardiah Binti Morsin, Chee Yen Leow and Akaa Agbaeze Etengusing, "Amplify-And-Forward Relay for Coverage Extension in Indoor Environments." Journal of Theoretical and Applied Information Technology, Vol.91. No.2, 30th September 2016.

[4] T. M. Cover and A. E. Gamal, "Capacity Theorems for The Relay Channel”, IEEE Trans. On Information Theory, Vol. 25, No. 5, Pp. 572584, Sept. 1979
[5] X. B. Yu, S. H. Leung, and W. K. Wong, "Performance analysis of variable-power adaptive modulation with antenna selection over Rayleigh fading channels," IET Communication., vol. 5, Iss. 10, pp. 1394-1404, Jul. 2011.

[6] Wei Lin et al., "Performance Analysis of Cooperative Networks with Random Decode-andForward Relaying," Proc. 10th IEEE Int. Conf. High Performance Computer. and Communication, Dalian, pp. 526-531, Sept. 2008.

[7] M. Sterna, et al., "Towards systems beyond 3G based on adaptive OFDMA transmission," Proc. IEEE, vol. 95, Iss. 12, pp. 2432-2455, Dec. 2007.

[8] S. H. O. Salih and M. M. A. Suliman, "Implementation of adaptive modulation and coding techniques using Matlab," Proc. 53rd International Symposium ELMAR-2011, Zadar, Croatia, pp. 137-139, 14-16 September 2011

[9] D. Morales-Jimenez, G. Gomez, J. F. Paris and J. T. Entrambasaguas, "Joint Adaptive Modulation and MIMO Transmission for Non-Ideal OFDMA Cellular Systems," IEEE Globecom Workshops, University of Malaga, Spain, pp. 1-5, 2009.

[10] X. Yu, S. H. Leung, W. H. Mow and W. K. Wong, "Performance of Variable-Power Adaptive Modulation with Space Time Coding and Imperfect CSI in MIMO Systems," IEEE Trans. Veh. Technol., vol. 58, Iss. 4, pp. 2115-2120, May 2009.

[11] A. Tesegaye, "Adaptive Modulation based OFDM System in Doubly Selective Channel," MSc. thesis, Dept. Elect. Eng and Comp., University of Addis Ababa, Ethiopia, 2012.

[12] E. Yohannes, "Adaptive Modulation-based MIMO systems for fading channels in LTE," MSc. thesis, Dept. Elect. Eng and Comp., University of Addis Ababa, Ethiopia, 2012.

[13] R.O. Abolade and Z. k Adeyemo, "Adaptive Modulation Performance In MIMO- OSTBC Over Rayleigh Fading Channel," Int. J. Eng. \& Computer Science, vol.12, Iss. 6, Apr. 2013.

[14] E. C. Strinati, S. Yang and J. C. Belfiore, "Adaptive Modulation and Coding for Hybrid Cooperative Networks," Int. Conf. Communications, Glasgow, pp. 4191-4195, 2007. 